Tropical Journal of Pharmaceutical Research March 2021; 20 (3): 649-659

ISSN: $1596-5996$ (print); 1596-9827 (electronic)

(C) Pharmacotherapy Group, Faculty of Pharmacy, University of Benin, Benin City, 300001 Nigeria.

\title{
Knowledge, awareness and practices regarding dietary supplements in Jordan
}

\author{
Haneen A Basheer ${ }^{1 *}$, Lina Elsalem², Deema Jaber ${ }^{1}$, Shorouk M Ibraheem¹, \\ Hamza Alhamad', Ahmad A Jum'ah ${ }^{3}$ \\ ${ }^{1}$ Faculty of Pharmacy, Zarqa University, PO Box 132222, Zarqa 13132, ${ }^{2}$ Department of Pharmacology, Faculty of Medicine, \\ ${ }^{3}$ Department of Conservative Dentistry, Faculty of Dentistry, Jordan University of Science and Technology, Irbid 22110, Jordan \\ *For correspondence: Email: hbasheer@zu.edu.jo; Tel: 00962-533821100 ext 1441; Fax: 00962-53821120
}

\begin{abstract}
Purpose: To investigative the knowledge, attitudes and beliefs pertaining to dietary supplements (DS) use in Jordan.

Methods: Participants were enrolled from four main cities in Jordan, viz, Amman, Zarqa, Irbid, and Mafraq, to fill a self-administered questionnaire which explored the objectives of the study.

Results: Most participants (80\%) knew what DS are. The prevalence of DS use was high (62.1\%) and significantly higher among females $(p=0.004)$. The most commonly consumed DS among users were in the form of multivitamins (93.0\%), vitamin $D(65.8 \%)$ and vitamin $C$ (57.6\%). About half of the participants (46.2\%) believed that DS have no side effects. The most frequent source of information for participants about DS was from non-reliable references, such as social media. Self-prescription was the most common approach by DS users for supplement intake. Finally, the common hypothesis of associating healthy habits with DS use was not found to be true among Jordanians.

Conclusion: These results highlight the need to implement appropriate awareness programs for the public regarding DS benefits and hazards, and especially to advise the public not to initiate DS use without first consulting an appropriate healthcare professional.
\end{abstract}

Keywords: Dietary supplements, Vitamins, Self-prescription

\begin{abstract}
This is an Open Access article that uses a fund-ing model which does not charge readers or their institutions for access and distributed under the terms of the Creative Commons Attribution License (http://creativecommons.org/licenses/by/4.0) and the Budapest Open Access Initiative (http://www.budapestopenaccessinitiative.org/read), which permit unrestricted use, distribution, and reproduction in any medium, provided the original work is properly credited.
\end{abstract}

Tropical Journal of Pharmaceutical Research is indexed by Science Citation Index (SciSearch), Scopus, International Pharmaceutical Abstract, Chemical Abstracts, Embase, Index Copernicus, EBSCO, African Index Medicus, JournalSeek, Journal Citation Reports/Science Edition, Directory of Open Access Journals (DOAJ), African Journal Online, Bioline International, Open-J-Gate and Pharmacy Abstracts

\section{INTRODUCTION}

The use of DS to treat health conditions is well established, with the use of vitamin $C$ to treat scurvy disease and vitamin $D$ in preventing and treating rickets as primary examples [1].

In addition, the role of DS in non-communicable diseases including cancer and cardiovascular complications has shown to be conflicting. It has been reported by many studies that DS can reduce the risk of developing the aforementioned health issues [1]. In contrast, some researchers have presented that the unnecessary use of DS showed neither positive nor negative outcomes, likewise, others have observed overwhelming side effects. In the Park et al study, an association between the use of multivitamins and the increase or decrease of cancer or cardiovascular deaths could not be found during 
the eleven years follow up study with 182,099 participants [2].

In recent years, there has been a substantial increase in the use of DS worldwide due to media advertising supplements as health products free of any potential risks. Accordingly, many studies have been conducted in different countries including Italy, Korea, and USA, to assess the population's knowledge about DS and to assure that they are used safely and effectively [3-5]. In Jordan, to our best knowledge, there is only one study that has examined the attitudes of DS users. However, it was only conducted among university students at University of Jordan; in which $27.4 \%$ of participants reported the use of DS mostly in the form of multivitamins [6].

Furthermore, there is an increasing evidence of an association between healthy habits such as regular physical activity, avoiding smoking products and maintaining healthy body weight with DS intake [7].

Limited evidence is available regarding the user's knowledge and attitudes among the Jordanian population, in respect to DS consumption in different demographic backgrounds. This study aims to investigate the awareness and knowledge about DS among the Jordanian population as well as their habits and beliefs regarding the use of supplements. The findings of the current study shed light on knowledge about DS use, factors influencing DS use, and the attitudes and characteristics of the typical user in Jordan.

\section{METHODS}

\section{Study design}

A cross sectional study was conducted in four main cities in Jordan including; Amman, Zarqa, Irbid, and Mafraq. Participants above 18 years old were randomly recruited during weekdays from individuals attending universities and workplaces over a period of six weeks from Nov 2019 , on a voluntary basis. The minimum required sample size (385) was calculated at $(95$ $\%)$ confidence level and (5\%) margin of error with $(50 \%)$ response [8]. The same research assistant handed all the self- administered questionnaires and a script containing information about the questionnaire and privacy rights was read to all participants beforehand. Persons who had no interest in the survey refused to participate and only the persons who agreed to the terms of participation were included. The informed written consent forms were obtained from all participants and all questionnaires were filled anonymously. The study protocol was conducted in accordance to the good clinical practices and principles as stated in the Declaration of Helsinki document [9]. The study was approved by the ethical committee review board at Zarqa University in Jordan (no: 3/1/2019-2020).

\section{Study tools}

The questionnaire was adapted after comprehensive literature search based on other relevant published questionnaires $[3,10]$. It was developed into closed/open ended questions and reviewed internally at the faculty of pharmacy at Zarqa University. Then, a pilot study of 60 participants was carried out to evaluate the clarity of the survey items as well as the analysis of preliminary data before performing the full launch. Data obtained during the internal piloting study was included in the main study analysis. The survey was designed in Arabic language and consisted of three main sections; the first section aimed to collect demographic characteristics; which included nationality, gender, age, educational level and discipline, social status, occupation, weight, height, smoking status and physical activity (frequency and intensity). The second part aimed to assess the participant's knowledge and awareness about DS. Participants were explicitly asked about the knowledge indicated by being able to give one example of DS, source of information, beliefs in term of benefits and risks regarding DS use. If the participants assumed a beneficial role of DS in the course of any disease, they were requested to indicate if DS have a role in terms of treatment and/or prevention of diseases and to specify the supplement involved in this role. Finally, the participants were asked in the third section to answer questions regarding their use of DS, reasons for intake, most consumed DS, prescription method, as well as their attitudes toward use of DS including adherence. Participants were allowed to choose more than one answer when appropriate. Participants were considered non-users of DS if they indicated that they had never used DS until the time of study.

\section{Data analysis}

Data were coded and inputted into IBM Statistical Package for Social Sciences (SPSS ${ }^{\odot}$ ) Statistics for Windows, Version 22, (IBM Corp., Armonk, N.Y., USA), for statistical analysis. Descriptive statistics with corresponding $95 \%$ confidence intervals were constructed. Differences between various groups were evaluated using Pearson chi-Square correlation test $X^{2}$ and Fisher exact 
tests for categorical variables. $P<0.05$ were considered statistically significant. To measure the association between variables, the Cramer's $\checkmark$ coefficient test was used. Results of Cramer's $\mathrm{V}$ coefficient test was interpreted as $>0.25=$ very strong, $>0.15=$ Strong, $>0.10=$ moderate, $>0.05=$ weak and $>0=$ no or very weak following "User's guide to correlation coefficients" by Akoglu [11].

\section{RESULTS}

Study sample and demographic
characteristics

Questionnaires were handed out to the public to participate in the survey entitled "Knowledge, awareness and practices about dietary supplements in Jordan". The final sample size was 509 participants. The participants were included in the study if they completed the questionnaire and gave written informed consent for participation.

The majority of participants were female (52.7 $\%$ ), in the age group 18 - 25 years $(45.0 \%)$, Jordanian (91.8\%), non-smokers (66\%), with abnormal weight as indicated by BMI (57.6\%) and physically inactive (54.6\%). In addition, most of the participants were bachelor degree holders $(63.3 \%)$ and have no association with the medical field (65.4\%) (Table 1 and 2$)$.

Table 1: Demographic characteristics of the study population

\begin{tabular}{lc}
\hline Demographics & $\begin{array}{c}\text { Sample size } \\
\mathrm{N}=\mathbf{5 0 9}(\%)\end{array}$ \\
$\begin{array}{l}\text { Age } \\
18-25 \text { years. }\end{array}$ & $\mathrm{N}=229(45.0)$ \\
$26-30$ years. & $\mathrm{N}=109(21.4)$ \\
$31-40$ years. & $\mathrm{N}=105(20.6)$ \\
$>40 y e a r s$. & $\mathrm{N}=66(13.0)$ \\
Gender & $\mathrm{N}=241(47.3)$ \\
Male & $\mathrm{N}=268(52.7)$ \\
Female & $\mathrm{N}=283(55.6)$ \\
Marital status & $\mathrm{N}=226(44.4)$ \\
$\begin{array}{l}\text { Single } \\
\text { Married }\end{array}$ & $\mathrm{N}=207(40.7)$ \\
Having Children & $\mathrm{N}=302(59.3)$ \\
$\begin{array}{l}\text { No } \\
\text { Nationality } \\
\text { Jordanian }\end{array}$ & $\mathrm{N}=467(91.8)$ \\
$\begin{array}{l}\text { Others } \\
\text { Education level } \\
\text { High school or less. }\end{array}$ & $\mathrm{N}=42(8.2)$ \\
$\begin{array}{l}\text { Bachelor. } \\
\text { Post graduate university } \\
\text { degree (e.g., MSc., PhD) }\end{array}$ & $\mathrm{N}=137(26.9)$ \\
\hline
\end{tabular}

Table 2: Demographic characteristics of the study

\begin{tabular}{|c|c|}
\hline Demographics & $\begin{array}{l}\text { Study population } \\
\quad N=509(\%)\end{array}$ \\
\hline \multicolumn{2}{|l|}{ Occupation } \\
\hline Student & $\mathrm{N}=175(34.4)$ \\
\hline Housewife & $N=68(13.4)$ \\
\hline Medical services & $\mathrm{N}=82(16.1)$ \\
\hline Private occupation & $\mathrm{N}=179(35.2)$ \\
\hline Others & $N=5(1.0)$ \\
\hline \multicolumn{2}{|l|}{$\begin{array}{l}\text { Medical field association } \\
\text { (students or workers) }\end{array}$} \\
\hline $\begin{array}{l}\text { Yes (Medicine, pharmacy, nursing } \\
\text { and others) }\end{array}$ & $N=176(34.6)$ \\
\hline No & $\mathrm{N}=333(65.4)$ \\
\hline \multicolumn{2}{|r|}{ ) } \\
\hline Underweight $\left(\mathrm{BMl}<18.5 \mathrm{~kg} / \mathrm{m}^{2}\right)$ & $\mathrm{N}=18(3.5)$ \\
\hline $\begin{array}{l}\text { Normal weight (BMI: } 18.5-24.9 \\
\mathrm{~kg} / \mathrm{m}^{2} \text { ) }\end{array}$ & $N=216(42.4)$ \\
\hline Overweight (BMI: $25-29.9 \mathrm{~kg} / \mathrm{m}^{2}$ ) & $N=185(36.3)$ \\
\hline Obese $\left(\mathrm{BMI}>30.0 \mathrm{~kg} / \mathrm{m}^{2}\right)$ & $\mathrm{N}=90(17.7)$ \\
\hline \multicolumn{2}{|l|}{ Smoking } \\
\hline Yes & $\mathrm{N}=173(34.0)$ \\
\hline No & $\mathrm{N}=336(66.0)$ \\
\hline \multicolumn{2}{|l|}{ Physical activity } \\
\hline No & $N=278(54.6)$ \\
\hline Yes & $\mathrm{N}=231(45.4)$ \\
\hline \multicolumn{2}{|l|}{ Frequency of exercise } \\
\hline 1 & $N=26(5.1)$ \\
\hline 2 & $\mathrm{~N}=67(13.2)$ \\
\hline 3 & $\mathrm{~N}=67(13.2)$ \\
\hline 4 & $\mathrm{~N}=32(6.3)$ \\
\hline$\geq 5$ & $\mathrm{~N}=35(6.8)$ \\
\hline \multicolumn{2}{|l|}{$\begin{array}{l}\text { Duration of exercise session } \\
\text { les }\end{array}$} \\
\hline $30-45$ minutes & $N=143(28.0)$ \\
\hline $60-90$ minutes & $\mathrm{N}=77(15.1)$ \\
\hline More than 90 minutes & $\mathrm{N}=11(2.2)$ \\
\hline
\end{tabular}

\section{Knowledge and awareness about DS}

Table 3 shows results in regard to knowledge and awareness about DS, most participants (80 $\%$ knew what DS are and most of the information was obtained from the internet and social media web pages (45\%). A significant difference was observed between genders (Table 4).

Females had more knowledge about DS $(p=$ 0.003 ) compared to males. Furthermore, the knowledge about DS was significantly higher among the medical field participants $(p=0.016)$ (Table 4).

Regarding possible side effects of DS, $46.8 \%$ of participants believed that DS have side effects (Table 3 ), and this was strongly associated with medical field specialty (Cramer's $V=0.184)(p=$ 0.005) (Table 4). Furthermore, participants who were physically active strongly believed that DS have side effects (Cramer's $V=0.184)(p=$ 0.001 , Table 4). In respect to the role of DS in disease prevention or treatment, the majority of 
participants answered that DS use could neither prevent $(64.6 \%)$ nor treat $(73.3 \%)$ chronic diseases (Table 3).

Table 3: Knowledge and awareness about DS among participants

\begin{tabular}{|c|c|}
\hline Do you know what DS are? & $\mathrm{N}=509,(\%)$ \\
\hline Yes & $\mathrm{N}=407(80.0)$ \\
\hline No & $\mathrm{N}=102(20.0)$ \\
\hline $\begin{array}{l}\text { What is your source of } \\
\text { information regarding DS?* }\end{array}$ & $\mathrm{N}=407,(\%)$ \\
\hline None & $\mathrm{N}=139(34.2)$ \\
\hline Physicians & $N=99(14)$ \\
\hline Pharmacists & $N=57(8.8)$ \\
\hline Social media and internet & $N=183(45)$ \\
\hline Family members & $\mathrm{N}=51(12.5)$ \\
\hline Sport Trainers & $\mathrm{N}=29(7.1)$ \\
\hline $\begin{array}{l}\text { Do you think that DS have } \\
\text { side effects? }\end{array}$ & $\mathrm{N}=509,(\%)$ \\
\hline Yes & $\mathrm{N}=238(46.8)$ \\
\hline No & $\mathrm{N}=235(46.2)$ \\
\hline I don't know & $\mathrm{N}=36(7.1)$ \\
\hline $\begin{array}{l}\text { Do you think that DS can } \\
\text { prevent chronic diseases? }\end{array}$ & $N=509,(\%)$ \\
\hline Yes & $\mathrm{N}=152(29.9)$ \\
\hline No & $\mathrm{N}=329(64.6)$ \\
\hline I don't know & $N=28(5.5)$ \\
\hline $\begin{array}{l}\text { Do you think that DS can treat } \\
\text { chronic diseases? }\end{array}$ & $\mathrm{N}=509,(\%)$ \\
\hline Yes & $\mathrm{N}=109(21.4)$ \\
\hline No & $\mathrm{N}=373(73.3)$ \\
\hline I don't know & $N=27(5.3)$ \\
\hline
\end{tabular}

This was found to be statistically significant, mainly among participants who are related to the medical field (Cramer's $V=0.178,0.131)(p \leq$ $0.005,0.013)$, respectively for prevention and treatment (Table 3).

Concerning gender difference, there was a strong association between gender and belief of DS role in disease course. Females believed significantly more in the role of DS in disease prevention and treatment (Cramer's $\mathrm{V}=0.157$, $0.158)(p=0.002,0.002)$, respectively (Table 4). Unexpectedly, most of the participants, even the ones who agreed that DS could prevent or treat diseases, were not sure about which diseases are treatable or preventable (Table 5).

Attitudes, habits and use of dietary
supplements

Most of the survey participants (62.1\%) have used DS, with the majority of DS users being females $(p=0.004)$ and from the age group 3140 years $(p=0.025)$. Furthermore, the belief about DS benefits was statistically significant among females $(p=0.013)$, medical field participants $(p=0.001)$ (Table 6).
In respect of association of DS intake and healthy habits, no association was found between DS use and BMI (Cramer's V =0.072), physical activity (Cramer's $\mathrm{V}=0.025)$, and smoking status (Cramer's V = 0.012) (Table 7).

The most commonly used DS by participants were in the form of multivitamins (93.0 \%) followed by vitamin D $(65.8 \%)$ and vitamin C $(57.6 \%)$ (Table 8). Of note, a very strong association was only found between the intake of body building supplements, such as proteins and creatine, with gender (Cramer's $V=0.286)$, and physical activity (Cramer's $V=0.252)(p \leq$ 0.005).

Furthermore, smoking status (Cramer's V = 0.223 ) and young age 18 - 25 years (Cramer's $V$ $=0.226$ ) were also strongly associated with body building supplements intake (Table 9). Other demographic data showed low or no association with body building supplements intake.

The study's results showed a very significant association between knowledge and use (Cramer's V $=0.408)(p \leq 0.005)$ (Table 10). Users believed more significantly than non-users that DS have no side effects, having a positive role in disease prevention or treatment. This observation was found statistically significant (Cramer's V > 0.2) ( $p \leq 0.005)$.

Regarding DS indications, the DS users mostly took DS to overcome a deficiency (51\%) and/or to improve their health and well-being (44\%). DS was mainly self-prescribed by the users $(92.1 \%)$ or prescribed by physicians (53.2 \%). The majority of users $(71.8 \%)$ were adherent to the recommended daily dose as indicated by the label or health care professionals.

\section{DISCUSSION}

Limited evidence is available regarding DS use among Jordanian population. One study was published by Suleiman et al about the vitaminmineral use in Jordan, however, it was only conducted on university students and from the age group 17 - 28 years old at university of Jordan [6]. To the best of our knowledge this study is the first to investigate the knowledge, awareness, beliefs and attitudes about use of DS by the Jordanian public. The results showed that the majority of participants (80\%) knew what DS are. This was significantly high among the medical field participants. This agrees with many studies that compare medical and non-medical participants' knowledge about DS [12]. 
Table 4: Knowledge and beliefs about DS among gender, medical field and physical activity groups

\begin{tabular}{|c|c|c|c|c|c|c|c|c|c|}
\hline \multirow[t]{2}{*}{ Variable } & \multirow[b]{2}{*}{ male } & \multicolumn{2}{|l|}{ Gender } & \multicolumn{3}{|c|}{ medical field affiliation } & \multicolumn{3}{|c|}{ Physical activity } \\
\hline & & female & total & Yes & No & total & active & inactive & total \\
\hline \multicolumn{10}{|c|}{ Do you know what DS are? } \\
\hline Yes, $\mathbf{N}$ & 179 & $228^{* *}$ & 407 & $152^{*}$ & 255 & 407 & 187 & 220 & 407 \\
\hline Column \% & 74.3 & 85.1 & 80 & 86.9 & 76.6 & 80 & 82.4 & 78 & 80 \\
\hline Column \% & 25.7 & 14.9 & 20 & 13.1 & 23.7 & 20 & 17.6 & 22 & 20 \\
\hline Total & 241 & 268 & 509 & 175 & 333 & 509 & 227 & 282 & 509 \\
\hline \multicolumn{10}{|c|}{ Do you think DS have adverse effects? } \\
\hline Yes, $\mathbf{N}$ & 116 & 122 & 238 & $102^{\star *}$ & 137 & 239 & $129^{* *}$ & 109 & 238 \\
\hline Column \% & 48.1 & 45.5 & 46.8 & 58.3 & 41 & 47 & 56.8 & 38.7 & 46.8 \\
\hline No, $\mathbf{N}$ & 106 & 129 & 235 & 68 & 166 & 235 & 85 & 150 & 235 \\
\hline Column \% & 44 & 48.1 & 46.2 & 39.0 & 49.7 & 46.2 & 37.4 & 53.2 & 46.2 \\
\hline I don't know, N & 19 & 17 & 36 & 5 & 30 & 35 & 13 & 23 & 36 \\
\hline Column \% & 7.9 & 6.3 & 7.1 & 2.9 & 9 & 6.9 & 5.7 & 8.2 & 7.1 \\
\hline Total & 241 & 268 & 509 & 175 & 334 & 509 & 227 & 282 & 509 \\
\hline \multicolumn{10}{|c|}{ Do you think DS can prevent diseases? } \\
\hline Yes, N & 59 & $93^{* *}$ & 151 & $65^{\star *}$ & 87 & 151 & 69 & 83 & 151 \\
\hline Column \% & 24.5 & 34.7 & 29.7 & 36.9 & 26.1 & 29.7 & 30.4 & 29.4 & 29.7 \\
\hline No, $\mathbf{N}$ & 162 & 168 & 329 & 110 & 220 & 329 & 147 & 183 & 329 \\
\hline Column \% & 67.2 & 62.7 & 64.6 & 62.5 & 66.2 & 64.6 & 64.8 & 64.9 & 64.6 \\
\hline I don't know, N & 20 & 7 & 29 & 1 & 26 & 29 & 11 & 16 & 29 \\
\hline Column \% & $\begin{array}{l}8.3 \\
7 \%\end{array}$ & 2.6 & 5.7 & 0.6 & 7.8 & 5.7 & 4.8 & 5.7 & 5.7 \\
\hline Total & 241 & 268 & 509 & 176 & 333 & 509 & 227 & 282 & 509 \\
\hline \multicolumn{10}{|c|}{ Do you think DS can treat diseases? } \\
\hline Yes, $\mathbf{N}$ & 43 & $66^{* *}$ & 109 & $45^{*}$ & 64 & 109 & 51 & 58 & 109 \\
\hline Column \% & 17.8 & 24.6 & 21.4 & 25.6 & 19.2 & 21.4 & 22.5 & 20.6 & 21.4 \\
\hline No, $\mathbf{N}$ & 177 & 196 & 373 & 128 & 245 & 373 & 164 & 209 & 373 \\
\hline Column \% & 73.4 & 73.1 & 73.3 & 72.7 & 73.6 & 73.3 & 72.2 & 74.1 & 73.3 \\
\hline I don't know, N & 21 & 6 & 27 & 3 & 24 & 27 & 12 & 15 & 27 \\
\hline Column \% & 8.7 & 2.2 & 5.3 & 1.7 & 7.2 & 5.3 & 5.3 & 5.3 & 5.3 \\
\hline Total & 241 & 268 & 509 & 176 & 333 & 509 & 227 & 282 & 509 \\
\hline
\end{tabular}


Table 5: Beliefs of participants regarding the role of DS in diseases or clinical complications

\begin{tabular}{|c|c|c|c|c|c|}
\hline $\begin{array}{l}\text { Disease or clinical } \\
\text { complications }\end{array}$ & $\begin{array}{l}\text { Prevention } \\
\text { N (\%) }\end{array}$ & $\begin{array}{l}\text { Treatment } \\
\text { N (\%) }\end{array}$ & $\begin{array}{c}\text { Prevention and treatment } \\
\text { N (\%) }\end{array}$ & $\begin{array}{l}\text { I don't Know } \\
\text { N (\%) }\end{array}$ & Examples on DS given by participants \\
\hline Pregnancy Complications & $68(13.4)$ & $12(2.4)$ & $52(10.2)$ & $377(74.1)$ & Iron, B complex, Folic acid, Calcium, Omega 3 \\
\hline Osteoporosis & $61(12.0)$ & $18(3.5)$ & $65(12.8)$ & $365(71.7)$ & Calcium, Vitamin D \\
\hline Osteoarthritis & $63(12.4)$ & $17(3.3)$ & $49(9.6)$ & $379(74.5)$ & Calcium, Vitamin D, Glucosamine, B complex \\
\hline Cancer & $68(13.4)$ & $9(1.8)$ & $39(7.7)$ & $393(77.2)$ & Vitamin D, B complex, Ginger, Garlic, Vitamin C\&E, Omega \\
\hline Cardiovascular Diseases & $67(13.2)$ & $18(3.5)$ & $45(8.8)$ & $378(74.3)$ & B complex, Ginger, Vitamin E, Cod liver oil, Zinc, Omega 3 \\
\hline Immunity & $61(12.0)$ & $10(2.0)$ & $60(11.8)$ & $378(74.3)$ & B complex, Vitamin C\&E, Omega 3 \\
\hline Influenza & $50(9.8)$ & $17(3.3)$ & $67(13.2)$ & $375(73.7)$ & Vitamin C, Ginger, Zinc \\
\hline Cataract (disambiguation) & $54(10.6)$ & $19(3.7)$ & $33(6.5)$ & $403(79.2)$ & B complex, Vitamin A, Vitamin D, Zinc \\
\hline Kidney disease & $56(11.0)$ & $9(1.8)$ & $40(7.9)$ & 404 (79.4) & Calcium, Vitamin D \\
\hline Diabetes mellitus & $61(12.0)$ & $14(2.8)$ & $42(8.3)$ & $492(77.0)$ & B complex, Garlic, Vitamin D, Fish oil \\
\hline
\end{tabular}

Table 6: Use and beliefs about DS among participants according to gender, age and medical affiliation

\begin{tabular}{|c|c|c|c|c|c|c|c|c|c|c|c|}
\hline \multirow[t]{2}{*}{ Variable } & \multicolumn{3}{|c|}{ Gender } & \multicolumn{5}{|c|}{ Age (years) } & \multicolumn{3}{|c|}{ Medical field affiliation } \\
\hline & Male & Female & Total & $18-25$ & $26-30$ & $31-40$ & $>40$ & Total & Yes & No & Total \\
\hline \multicolumn{12}{|c|}{ Have you used DS? } \\
\hline Yes, $\mathbf{N}$ & 134 & $182^{* *}$ & 316 & 130 & $67(21.2)$ & $78^{*}(24.7)$ & 41 & 316 & 117 & 199 & 316 \\
\hline Column \% & 55.6 & 68.0 & 62.1 & 57.3 & 61.5 & 74.3 & 60.3 & 62.1 & 66.5 & 59.8 & 62.1 \\
\hline No, $\mathbf{N}$ & 107 & 86 & 193 & $99(50.3)$ & $42(21.8)$ & 27 & 25 & 193 & 59 & 134 & 193 \\
\hline Column \% & 44.4 & 32.1 & 37.9 & 43.2 & 38.5 & 25.7 & 37.7 & 37.9 & 33.5 & 40.2 & 37.9 \\
\hline Total & 241 & 268 & 509 & $229(45)$ & $109(21.4)$ & $105(21)$ & 66 & 509 & 176 & 333 & 509 \\
\hline \multicolumn{12}{|c|}{$\begin{array}{l}\text { Do you believe that DS have } \\
\text { benefits? }\end{array}$} \\
\hline Yes, $N$ & 175 & $215^{*}$ & 390 & 167 & 84 & 88 & 51 & 390 & $150^{* *}$ & 240 & 390 \\
\hline Column \% & 72.6 & 80.2 & 76.6 & 72.9 & 77.1 & 88.3 & 77.3 & 76.6 & 85.2 & 72.1 & 76.6 \\
\hline No, $\mathbf{N}$ & 48 & 47 & 95 & 51 & 18 & 13 & 13 & 95 & 24 & 71 & 95 \\
\hline Column \% & 19.9 & 17.5 & 18.6 & 22.3 & 16.5 & 12.4 & 19.7 & 18.7 & 13.6 & 21.3 & 18.7 \\
\hline I don't know, N & 18 & 6 & 24 & $11(45.8)$ & $7(29.2)$ & 4 & 2 & 24 & 2 & 22 & 24 \\
\hline Column \% & 7.5 & 2.2 & 4.7 & 4.8 & 6.4 & 3.8 & 2.9 & 4.7 & 1.1 & 6.6 & 4.8 .7 \\
\hline Total & 241 & 268 & 509 & 229 & 109 & 105 & $66(13)$ & 509 & 176 & 333 & 509 \\
\hline
\end{tabular}

${ }^{* *} P$ value $\leq 0.005,{ }^{*} P$ value $\leq 0.05 . D S=$ Dietary Supplements 
Table 7: Association between DS use and indicators of healthy habits

\begin{tabular}{|c|c|c|c|c|c|c|c|c|c|c|c|}
\hline \multirow[t]{2}{*}{ Variable } & \multicolumn{5}{|c|}{ BMI } & \multicolumn{3}{|c|}{ Physical activity } & \multicolumn{3}{|c|}{ Smoking } \\
\hline & $<18.5$ & $18.5-24.9$ & $25-30$ & $>\mathbf{3 0}$ & Total & Active & Inactive & Total & Yes & No & Total \\
\hline \multicolumn{12}{|c|}{ Have you used DS? } \\
\hline Yes, N & 9 & 137 & 116 & 54 & 316 & 144 & 172 & 316 & 106 & 210 & 316 \\
\hline Column \% & 50 & 63.4 & 62.7 & 60.0 & 62.1 & 63.4 & 61 & 62.1 & 61.3 & 62.5 & 62.1 \\
\hline Row \% & 2.8 & 43.4 & 36.7 & 17.1 & 100.0 & 45.6 & 54.4 & 100.0 & 33.5 & 66.5 & 100.0 \\
\hline No, $\mathbf{N}$ & 9 & 79 & 69 & 36 & 193 & 83 & 110 & 193 & 67 & 126 & 193 \\
\hline Column \% & 50 & 36.6 & 37.3 & 40.0 & 37.9 & 36.5 & 39 & 37.9 & 38.7 & 37.5 & 37.9 \\
\hline Row \% & 4.7 & 40.9 & 35.8 & 18.7 & 100.0 & 43.0 & 57.0 & 100.0 & 34.7 & 65.3 & 100.0 \\
\hline Total, $\mathbf{N}$ & 18 & 216 & 185 & 90 & 509 & 227 & 282 & 509 & 173 & 336 & 509 \\
\hline \multicolumn{12}{|c|}{ Do you believe that DS have benefits? } \\
\hline Yes, $\mathbf{N}$ & 13 & 167 & 141 & 69 & 390 & 179 & 211 & 390 & $136^{*}$ & 254 & 390 \\
\hline Column \% & 72.2 & 77.3 & 76.2 & 76.7 & 76.6 & 78.9 & 74.8 & 76.6 & 78.6 & 75.6 & 76.6 \\
\hline Row \% & 3.3 & 42.8 & 36.2 & 17.7 & 100.0 & 45.9 & 54.1 & 100.0 & 34.9 & 65.1 & 100.0 \\
\hline No, $\mathbf{N}$ & 4 & 38 & 36 & 17 & 95 & 40 & 55 & 95 & 24 & 71 & 95 \\
\hline Column \% & 22.2 & 17.6 & 19.5 & 18.9 & 18.7 & 17.6 & 19.5 & 18.7 & 13.9 & 21.1 & 18.7 \\
\hline Row \% & 4.2 & 40.0 & 37.9 & 17.9 & 100 & 42.1 & 57.9 & 100.0 & 25.3 & 74.7 & 100.0 \\
\hline $\begin{array}{l}\text { I don't know, } \\
\mathrm{N}\end{array}$ & 1 & 11 & 8 & 4 & 24 & 8 & 16 & 24 & 13 & 11 & 24 \\
\hline Column \% & 5.6 & 5.1 & 4.3 & 4.4 & 4.7 & 3.5 & 5.7 & 4.7 & 7.5 & 3.3 & 4.7 \\
\hline Row \% & 4.2 & 45.8 & 33.3 & 16.7 & 100 & 33.3 & 66.7 & 100.0 & 54.2 & 45.8 & 100.0 \\
\hline Total, $\mathbf{N}$ & 18 & 216 & 185 & 90 & 509 & 227 & 282 & 509 & 173 & 336 & 509 \\
\hline
\end{tabular}

${ }^{*} P$ value $\leq 0.05$. Carmer's $V$ test showed no association between $D S$ intake and indicators of healthy habits (BMI, Physical activity and being a non-smoker). Carmer's $V$ values $<0.05 . D S=$ Dietary Supplements 
Table 8: The most common consumed DS by users

\begin{tabular}{llll}
\hline Type of DS used & $\begin{array}{l}\text { Male } \\
\mathbf{N}(\%)\end{array}$ & $\begin{array}{l}\text { Female } \\
\mathbf{N}(\%)\end{array}$ & $\begin{array}{c}\text { Total } \\
\mathbf{N}(\%)\end{array}$ \\
\hline Multivitamins & & & $294(93.0)$ \\
Vitamin D & $118(40.1)$ & $176(59.9)$ & $208(65.8)$ \\
Vitamin C & $73(35.1)$ & $135(64.9)$ & $182(57.6)$ \\
Vitamin A & $76(41.8)$ & $106(58.20)$ & $83(26.3)$ \\
Vitamin B & $38(45.8)$ & $45(54.2)$ & $83(26.3)$ \\
Vitamin B complex & $40(48.2)$ & $43(51.8)$ & $73(23.1)$ \\
Vitamin E & $22(30.1)$ & $51(69.9)$ & $53(16.8)$ \\
Vitamin K & $25(47.2)$ & $28(52.8)$ & $32(10.1)$ \\
Body building supplements & $44(71)$ & $16(50)$ & $62(19.6)$ \\
Sport drinks & $16(50)$ & $18(29)$ & $45(14.2)$ \\
Weight loss products & $16(30.2)$ & $37(69.8)$ & $53(16.8)$ \\
Stimulants & $11(47.8)$ & $12(52.2)$ & $23(7.3)$ \\
Others (ginseng,ginko, etc.,) & $1(7.1)$ & $13(92.9)$ & $14(4.4)$ \\
\hline
\end{tabular}

${ }^{*}$ Participants were allowed to choose more than one answer as appropriate. DS = Dietary Supplements

Table 9: Association between body building supplements and demographic data

\begin{tabular}{|c|c|c|c|}
\hline & \multicolumn{3}{|c|}{$\begin{array}{l}\text { Proteins and body building supplements } \\
\text { intake }\end{array}$} \\
\hline & $\begin{array}{c}\text { Yes } \\
\mathrm{N}(\%)\end{array}$ & $\begin{array}{c}\text { No } \\
\mathrm{N}(\%)\end{array}$ & $\begin{array}{l}\text { Total } \\
\text { N(\%) }\end{array}$ \\
\hline $\begin{array}{l}\text { Gender } \\
\text { Male }\end{array}$ & $44^{* *}(32.8)$ & $90(67.2)$ & 134 \\
\hline Female & $18(9.9)$ & $164(90.1)$ & 182 \\
\hline Smoking & & & \\
\hline Yes & $\begin{array}{c}34^{* *}(32.1) \\
28\end{array}$ & $72(67.9)$ & 106 \\
\hline $\begin{array}{l}\text { No } \\
\text { Physical activity }\end{array}$ & $28(13.3)$ & $182(86.7)$ & 210 \\
\hline Yes & $44^{* *}(30.6)$ & $100(69.4)$ & 144 \\
\hline No & $18(13.9)$ & $154(89.5)$ & 172 \\
\hline Age & & & \\
\hline $18-25$ & 39 ** $(30)$ & $91(70)$ & 130 \\
\hline $26-30$ & $10(14.9)$ & 57 (85.1) & 67 \\
\hline $31-40$ & $10(12.8)$ & $68(87.2)$ & 78 \\
\hline$>40$ & $3(7.3)$ & $38(92.7)$ & 41 \\
\hline
\end{tabular}

${ }^{* *} P$ value $\leq 0.005$. Cramer's $V$ value $>0.25$ (very strong association) was reported for association of body building supplements intake with gender and physical activity while Cramer's $V$ value $>0.15$ (strong association) was reported for association with smoking and age. Only Demographics that have significant association with body building supplements are shown in the table 8 
Table 10: Association factors with DS use

\begin{tabular}{|c|c|c|c|}
\hline & User & Non-user & Total \\
\hline \multicolumn{4}{|c|}{ Knowledge about DS } \\
\hline Yes & $293^{* *}$ & 114 & 407 \\
\hline Column\% & 92.7 & 59.1 & 80.0 \\
\hline No & 23 & 79 & 102 \\
\hline Column \% & 7.3 & 40.9 & 20.0 \\
\hline Total & 316 & 193 & 509 \\
\hline \multicolumn{4}{|c|}{ Acknowledging DS side effects } \\
\hline Yes & 148 & 90 & 238 \\
\hline Column\% & 46.8 & 46.6 & 46.9 \\
\hline No & $159^{* *}$ & 76 & 235 \\
\hline Column \% & 50.3 & 39.4 & 46.2 \\
\hline I don't know & 9 & 27 & 36 \\
\hline Column\% & 2.8 & 14.0 & 7.1 \\
\hline Total & 316 & 193 & 509 \\
\hline \multicolumn{4}{|c|}{ Prevention of disease } \\
\hline Yes & $112^{* *}$ & 40 & 151 \\
\hline Column \% & 35.4 & 20.7 & 29.7 \\
\hline No & 202 & 127 & 329 \\
\hline Column \% & 63.9 & 65.8 & 64.6 \\
\hline I don't know & 2 & 26 & 29 \\
\hline Column \% & 0.6 & 13.5 & 5.7 \\
\hline Total & 316 & 193 & 509 \\
\hline \multicolumn{4}{|c|}{ Treatment of disease } \\
\hline Yes & $79^{* *}$ & 30 & 109 \\
\hline Column \% & 25.0 & 15.5 & 21.4 \\
\hline No & 235 & 138 & 373 \\
\hline Column \% & 74.4 & 71.5 & 73.3 \\
\hline I don't know & 2 & 25 & 27 \\
\hline Column \% & 0.6 & 13 & 5.3 \\
\hline Total & 316 & 193 & 509 \\
\hline
\end{tabular}

${ }^{* *} P$ value $\leq 0.005$. Cramer's $V$ value $>0.25$ (very strong association was reported for Association of DS intake with knowledge about $D S$ and role of $D S$ in disease prevention and treatment while Cramer's $V$ value $>0.15$ (strong association) was reported for association of DS use with the assumption of no side effects. DS = Dietary Supplements

Indeed, more than half of the participants have used DS and these users were mostly females from the age group 30-41 years old. In this regard, our results were in agreement with previous studies, in which the users where mostly females and from older age groups [13].

Many studies worldwide have shown that nonsmokers and physically active participants consumed DS at a higher rate [14]. On the contrary, this study results showed no association between using DS and positive habits such as regular exercise and nonsmoking. The difference between these reported results and others could be attributed to the high proportion of smokers and social acceptance of smoking in Jordan [15], with $33.5 \%$ of DS users in the current report being smokers. Furthermore, our results showed that exercise is not a regular habit by users as $54.4 \%$ were physically inactive and $53.8 \%$ of the users were with BMI indicating overweight or obesity. In fact, obesity and overweight are concerning issues in Jordan [16]. Altogether, the belief that DS users are more interested in improving overall health by maintaining healthy habits was shown to be untrue by our results and another Korean study [4].

Based on findings from this study and others [3], the reason(s) for the intake of DS were mainly to overcome a deficiency or to improve health and wellbeing. The most DS used were in the form of multivitamins followed by vitamin $D$ and/or vitamin $\mathrm{C}$. The benefits of DS use are well documented in case of nutrients deficiencies [1].

The use of DS by the participants to prevent or treat diseases was low. However, these results were expected since the majority of them did not believe in the role of DS in reducing the risk of diseases or treating them. Furthermore, they 
were mostly uneducated about which DS are involved in disease prevention and/or treatment. Few studies have shown that DS can reduce the risk of chronic diseases [17]. Nonetheless, others have reported that the use of DS to treat or prevent diseases in healthy individuals without nutrients deficiencies is questionable and might be a health concern [18]. For instance, the use of vitamin $E$ increased the risk of prostate cancer as shown in a follow-up study conducted on 35,533 men from the USA, Canada, and Puerto Rico for 4 years [19].

In regard to source of influence for DS intake, most of the participants of this study consumed DS by self-prescription, which might put them at risk of developing potential side effects. This selfprescription could be related to the fact that DS are advertised to be generally safe. Lam et al have reported similar results to our study, in which $84.4 \%$ users were using self-prescribed DS [20]. DS users in present survey either did not seek information on supplements or if they did it was mostly from non-reliable sources such as social media and friends rather than healthcare providers. Consistent with these results, Alfawaz et al have shown that social media was the most common source of information about DS [21].

Our results showed that about half of our DS users were not aware of potential side effects; this awareness was significantly higher among medical participants and physically active participants. Jovanov et al have shown in their international study that the majority of young athletes $(72.1 \%)$ who were using DS to enhance body image were aware of health risks associated with body building supplements use, nonetheless this did not stop them of using the supplements [22]. In reference to the usage of body building supplements, such as creatine and chained amino acids, our results showed higher intake among physically active participants and males. Therefore, advising physically active people about natural resource alternatives to enhance sports performance and body image is recommended to reduce the possible risks associated with the use of body building supplements. It is well documented that exceeding the normal required level of nutrients has its own risks. Many studies have shown that using DS in excess can result in a different range of side effects; as simple as diarrhea to a more serious risk of liver injury and cancer [5].

Based on current evidence of DS use with reference to its possible side effects, the public should always be encouraged to seek medical advice from trustworthy and reliable resources.
Therefore, supplement intake should not be initiated before health care providers' recommendations; based on results from biochemical laboratory assays. This is highly valuable to avoid any harmful effects that might result from exceeding the normal levels of vitamins and minerals.

\section{Limitations of the study}

The present study has some limitations that should be considered. Our findings were from participants from 4 major cities in Jordan and therefore to have better generalization of the results, further studies including other parts of Jordan should be included.

\section{CONCLUSION}

DS users are more likely to take supplements on their own instead of consulting healthcare providers. Consequently, when this selfrecommended use of DS is linked with their personal belief that DS are free from any side effects and can treat or prevent a disease, the risks of DS use might outweigh the benefits, leading to negative health consequences. Therefore, it is recommended that the public should be educated about the advantages and disadvantages of DS, by implementing educational programs mainly through healthcare providers as they are the most accessible professionals to the public and have more opportunities to make a significant impact in these settings.

\section{DECLARATIONS}

\section{Acknowledgement}

The authors thank Zarqa University for providing the facilities to carry out this research.

\section{Conflict of interest}

The authors declare that they have no conflict of interest with regard to this work.

\section{Contribution of authors}

We declare that this work was done by the authors named in this article and all liabilities pertaining to claims relating to the content of this article will be borne by the authors Each author has contributed to the work as follow: Haneen A. Basheer: Conceptualization, Methodology, Writing- Original draft preparation, Formal analysis, Software, Project administration, Supervision, Data curation, Visualization. Lina 
Elsalem: Writing- Original draft preparation, Methodology, Resources, Writing Review \& Editing, Visualization. Deema Jaber: Data curation, Formal analysis, Writing - Review \& Editing. Shorouk M. Ibraheem: Methodology Data entry, Data entry into SPSS, Writing Review \& Editing. Hamza: Alhamad: Methodology, Writing - Review \& Editing. Ahmad A Jum'ah: Visualization Writing - Review and Editing.

\section{Open Access}

This is an Open Access article that uses a funding model which does not charge readers or their institutions for access and distributed under the terms of the Creative Commons Attribution License (http://creativecommons.org/licenses/by/ 4.0) and the Budapest Open Access Initiative (http://www.budapestopenaccessinitiative.org/rea d), which permit unrestricted use, distribution, and reproduction in any medium, provided the original work is properly credited.

\section{REFERENCES}

1. Darnton-Hill I. Public Health Aspects in the Prevention and Control of Vitamin Deficiencies. Curr Dev Nutr 2019; 3(9): nzz075.

2. Park S-Y, Murphy SP, Wilkens LR, Henderson BE, Kolonel LN. Multivitamin use and the risk of mortality and cancer incidence: the multiethnic cohort study. Am J Epidemiol 2011; 173(8): 906-914.

3. del Balzo $V$, Vitiello $V$, Germani $A$, Donini LM, Poggiogalle E, Pinto A. A cross-sectional survey on dietary supplements consumption among Italian teenagers. J Endocrinol Invest 2014; 9(7): e100508.

4. Kim JW, Lee SH, Kim JE, Han KD, Kwack TE, Kim BS, Kim JE, Jo EB, Park YK, Lee KS. The Association between Taking Dietary Supplements and Healthy Habits among Korean Adults: Results from the Fifth Korea National Health and Nutritional Examination Survey (2010-2012). Korean J Fam Med 2016; 37(3): 182-187.

5. Ronis MJJ, Pedersen KB, Watt J. Adverse Effects of Nutraceuticals and Dietary Supplements. Annu Rev Pharmacol Toxicol 2018; 58: 583-601.

6. Suleiman AA, Alboqai OK, Yasein N, Al-Essa MK, El Masri K. Prevalence of vitamin-mineral supplement use among Jordan University students. Saudi Med J 2008; 29(9): 1326-1331.

7. Dickinson A, MacKay D. Health habits and other characteristics of dietary supplement users: a review. Nutr J 2014; 13: 14.

8. Bolarinwa OA. Sample size estimation for health and social science researchers: The principles and considerations for different study designs. Niger Postgrad Med J 2020; 27(2): 67-75.

9. Goodyear MDE, Krleza-Jeric K, Lemmens T. The Declaration of Helsinki. BMJ (Clinical research ed.) 2007; 335(7621): 624-625.

10. Sirico F, Miressi S, Castaldo C, Spera R, Montagnani S, Di Meglio F, Nurzynska D. Habits and beliefs related to food supplements: Results of a survey among Italian students of different education fields and levels. PLoS One 2018; 13(1): e0191424-e0191424.

11. Akoglu H. User's guide to correlation coefficients. Turk $J$ Emerg Med 2018; 18(3): 91-93.

12. Alhomoud FK, Basil M, Bondarev A. Knowledge, Attitudes and Practices (KAP) Relating to Dietary Supplements Among Health Sciences and Non-Health Sciences Students in One of The Universities of United Arab Emirates (UAE). J Clin Diagn Res 2016; 10(9): Jc05-jc09.

13. Alowais MA, Selim MAE. Knowledge, attitude, and practices regarding dietary supplements in Saudi Arabia. J Family Med Prim Care 2019; 8(2): 365-372.

14. Bailey RL, Gahche JJ, Lentino CV, Dwyer JT, Engel JS, Thomas PR, Betz JM, Sempos CT, Picciano MF. Dietary supplement use in the United States, 2003-2006. J Nutr 2011; 141(2): 261-266.

15. Abu-Helalah MA, Alshraideh $H A$, Al-Serhan $A A$, Nesheiwat Al, Da'na M, Al-Nawafleh A. Epidemiology, attitudes and perceptions toward cigarettes and hookah smoking amongst adults in Jordan. Environ Health Prev Med 2015; 20(6): 422-433.

16. Alarjan JF, Hindawi OS, Judge LW, Aleyadh ZA, Bellar $D M$. Prevalence of obesity and behaviors associated with the development of metabolic disease among medical practitioners in Jordan. J Educ Health Promot 2015; $4: 17$.

17. Walrand S. Dietary supplement intake among the elderly: hazards and benefits. Curr Opin Clin Nutr Metab Care 2018; 21(6): 465-470.

18. Moyer MW. Nutrition: vitamins on trial. Nature 2014; 510(7506): 462-464.

19. Long-term study finds vitamin $E$ supplements raise the risk of prostate cancer. Oncology (Williston Park) 2011; 25(12): 1236-1237.

20. Lam A, Bradley G. Use of self-prescribed nonprescription medications and dietary supplements among assisted living facility residents. J Am Pharm Assoc (2003) 2006; 46(5): 574-581.

21. Alfawaz $H$, Khan $N$, Alfaifi $A$, Shahrani $F M$, Al Tameem HM, Al Otaibi SF, Abudigin WI, Al-Shayaa MS, AlGhanim SA, Al-Daghri NM. Prevalence of dietary supplement use and associated factors among female college students in Saudi Arabia. 2017; 17(1): 116.

22. Jovanov $P$, Đorđić V, Obradović B, Barak O, Pezo L, Marić A, Sakač M. Prevalence, knowledge and attitudes towards using sports supplements among young athletes. Journal of the International Society of Sports Nutrition 2019; 16(1): 27.

Trop J Pharm Res, March 2021; 20(3): 659 